\title{
Carrier Extraction Dynamics from Ge/Si Quantum Wells in Si Solar Cells
}

Takeshi Tayagaki ${ }^{1,2}$, Yusuke Hoshi ${ }^{3}$, Kazufumi Ooi $^{1}$, Takanori Kiguchi ${ }^{4}$, and Noritaka Usami ${ }^{3}$

\author{
${ }^{1}$ Institute for Chemical Research, Kyoto University, Uji, Kyoto 611-0011, Japan \\ ${ }^{2}$ PRESTO-JST, Kawaguchi, Saitama 332-0012, Japan \\ ${ }^{3}$ Graduate School of Engineering, Nagoya University, Nagoya, Aichi 464-8603, Japan \\ ${ }^{4}$ Institute for Materials Research, Tohoku University, Sendai 980-8577, Japan
}

To address the carrier extraction mechanism that determines the fundamental characteristics, such as current density, open circuit voltage, and fill factor in nanostructure-based solar cells, we performed photoluminescence (PL) decay measurements of the Ge/Si quantum wells (QWs) in crystalline-silicon ( $c$-Si) solar cells. We found that the PL decay time of Ge/Si QWs depends on the temperature and the applied electric field; this dependence reflects the carrier separation characteristics of electron-hole pairs in Ge/Si QWs. Above $\sim 40 \mathrm{~K}$, the electron-hole pairs are rapidly separated by the thermal excitation and the built-in electric field of $c$-Si solar cells. In contrast, at $20 \mathrm{~K}$ the PL decay time remains almost unchanged for an applied electric field of up to $\pm 1 \mathrm{~V}$. These results indicate that the electrons confined in the type-II band offsets could be thermally excited and then extracted by an applied electric field. 


\section{Introduction}

To overcome the limit of conventional single-junction devices [1], solar cells using quantum dots (QDs) and quantum wells (QWs), which enable the optical absorption of photons with energies below the band gap of the host, have been extensively studied [2-4]. One of the most important problems in the solar cells using semiconductor nanostructures is understanding the carrier extraction mechanism, in which the carriers that are confined to the nanostructures are extracted as electricity. This carrier extraction mechanism determines the fundamental characteristics, such as current density, open circuit voltage, and fill factor in nanostructure-based solar cells. For example, recent research has pointed out that open circuit voltage reductions in QD-based intermediate-band-type solar cells can be caused by thermal carrier extraction from the QDs [5,6]. Moreover, in hot carrier type solar cells [7], the carrier extraction filter for a definite energy (called selective energy contact) is one of the critical components. QDs and QWs may be able to implement such a selective energy contact [8-10]. However, the carrier extraction dynamics from such semiconductor nanostructures in solar cells is still not well understood.

In our quest to study carrier extraction dynamics, the $\mathrm{Ge} / \mathrm{Si}$ heterostructures provide a unique opportunity to investigate the optical and electronic properties in artificially controlled nanostructures [11-14]. Incorporation of Ge QDs in the intrinsic region of crystalline-silicon (c-Si) solar cells offer one way to absorb a low-energy photon that is not absorbed by silicon [15-19]. The external quantum efficiency in the near-infrared region has thus far been found to increase in multi-stacked Ge QDs structures [15], by using photonic nanostructures [17], and under strong light irradiations [18]. In contrast to the island-like QDs that are formed on a Ge/Si layer due to the Stranski-Krastanov method, the role of the wetting layers for the carrier transport in the QD solar cells is still not well understood. There is particular confusion about 
the impact of a type-II band offset in the Ge/Si heterostructure, where electrons and holes are expected to be spatially separated by the heterostructures to reduce their radiative and nonradiative recombination [17].

In this paper, we investigate the photoluminescence (PL) decay dynamics under different temperatures and voltage applications and discuss the carrier extraction mechanism in $\mathrm{Ge} / \mathrm{Si}$ QWs in $c$-Si solar cells. From PL decay measurements, we found that electron-hole pairs in the QWs are rapidly separated by the built-in electric field in $c$-Si solar cells. The temperature dependence of the PL decay measurements indicates that electrons confined to the Si layer due to a type-II band offset play a primary role in this rapid carrier separation.

\section{Experimental details}

The sample solar cells as illustrated in Fig. 1(a) were grown using a gas-source molecular beam epitaxy system (Air-Water VCE S2020) utilizing disilane and germane as the gas sources on $p$-type $\mathrm{Si}(100)$ substrates with a resistivity of $0.8-1.2 \Omega \mathrm{cm}[20]$. Ge/Si QWs with a 2-monolayer nominal Ge thickness stacked in a 50-layer structure were separated by a 20-nm-thick Si spacer layer. A $p-i-n$ junction was formed via the thermal diffusion of phosphorous from a spin-coated diffusion source [17]. The electric field applied to Ge/Si QWs were tuned by an external bias voltage [Fig. 1(b)]. We performed dark current measurements with a computer-interfaced Keithley 2636A source measurement unit and PL decay measurements with the second harmonics of a Ti:sapphire laser $(3.1 \mathrm{eV})$ and a photomultiplier-equipped photon-counting system [21]. The excitation laser power was 1-5 $\mathrm{mW}$. 


\section{Results and Discussions}

Figure 2(a) shows the PL spectra of Ge/Si QWs in $c$-Si solar cells measured at 20 and 50 K. PL peaks appeared at $\sim 1135,1155,1200$, and $1280 \mathrm{~nm}$; these peaks correspond to the transverse-optical (TO) phonon-assisted transitions in $c$-Si, the no-phonon-assisted transition, and the TO and transverse-acoustic phonon-assisted transitions of $\mathrm{Ge} / \mathrm{Si} \mathrm{QWs}$, respectively [22]. At $50 \mathrm{~K}$, PL intensity decreased with respect to that at $20 \mathrm{~K}$, but it increased under forward bias application at $50 \mathrm{~K}$. Here, we confirmed that without photoexcitation, no photoemission have been observed even at the forward bias condition of $V=1 \mathrm{~V}$, meaning that the enhanced PL intensity does not originate from electroluminescence; thus, the enhanced PL is caused by the suppressed nonradiative carrier reduction under the electric field application. Note that at zero external bias electric field condition, the built-in voltage of $\sim 1 \mathrm{~V}$ is applied to the QWs, as discussed later.

Figure 2(b) shows current density $(J)$-voltage $(V)$ characteristics of the $c$-Si solar cell with Ge/Si QWs measured at 20, 50, and $300 \mathrm{~K}$. As temperatures dropped, the dark current decreased for both forward and reverse bias conditions. Even at $20 \mathrm{~K}$ the rectification property was observed, in which the dark current density under forward bias condition is more than two orders smaller than that at $50 \mathrm{~K}$. This indicates that the carrier transport becomes inefficient at low temperatures because of the carrier confinements at the $\mathrm{Ge} / \mathrm{Si}$ QWs.

To understand the recovery of the PL intensity as shown in Fig. 2(a), we performed PL decay measurements in the Ge/Si QW solar cells. Figure 3(a) shows the temporal changes in the PL intensity of Ge/Si QWs at zero bias condition measured at different temperatures. The PL intensity at $1200 \mathrm{~nm}$, as indicated by the arrow in Fig. 2(a), was used for the PL decay measurements because all the PL intensity of phonon assisted transition and no-phonon 
transitions reflect the same carrier population. The PL decay time that is obtained with a single exponential function is summarized as a function of temperature in the inset of Fig. 3(a). The PL decay time decreased with increasing temperature. We confirmed that the PL decay rate is independent of the excitation laser power, demonstrating the negligibility of the nonradiative Auger recombination $[13,21]$. In combination with the reduced PL intensity, these behaviors indicate that the electron-hole pairs at QWs should be reduced by a nonradiative channel, such as an electron-hole separation. Figure 3(b) shows a summary of the temperature dependence of the PL decay rate, which, at high temperatures, indicates the promoted carrier reduction. Here, we considered the carrier extraction from QWs that occurred by thermal activation processes, radiative recombination, and nonradiative recombination in QWs. Consequently, the decay rate is given as follows:

$$
\Gamma_{\text {decay }}=\gamma_{0} \exp \left(-\frac{E_{a}}{k_{B} T}\right)+\gamma_{\text {rad }}+\gamma_{\text {nonrad }}
$$

where $\gamma_{0}, E_{a}, \gamma_{\text {rad }}$, and $\gamma_{\text {nonrad }}$ are the carrier extraction rate, activation energy, radiative recombination rate, and nonradiative recombination rate, respectively. Using Eq. (1) as shown in Fig. 3(b), we obtained $E_{a}=33 \mathrm{meV}, \gamma_{0}=1800 \mathrm{~ns}^{-1}$, and $\gamma_{\text {rad }}+\gamma_{\text {nonrad }}=0.13 \mathrm{~ns}^{-1}$, respectively. The total recombination rate $\gamma_{\text {rad }}+\gamma_{\text {nonrad }}$ is a little larger than the PL decay rate in the as-grown Ge/Si QWs $\left(<0.1 \mathrm{~ns}^{-1}\right)$. This may be related to the modification of electronic structure in the Ge/Si QWs during thermal annealing for the $p-i-n$ junction formation $[19,21]$. The obtained activation energy $E_{a}$ is consistent with the temperature dependence of dark current density, as shown in Fig. 2(b), in which the dark current at reverse bias condition significantly decreased below $50 \mathrm{~K}$. The inset in Fig. 3(b) illustrates a carrier transportation of electrons and holes in Ge/Si QWs in $c$-Si solar cells. Due to the type-II band offset, the electrons and hole are confined to the $\mathrm{Si}$ and Ge layers, respectively. The activation energy 
should then be related to the band offset energy of the electrons rather than the holes because the confinement depth of holes is much larger than that of electrons [23]. This means that in the Ge/Si QW solar cells the electrons should play a more dominant role in the carrier transport than holes; it also indicates that at room temperature electrons can freely move across the Ge/Si QWs layers even though the $\mathrm{Ge} / \mathrm{Si}$ interfaces may reduce the carrier diffusion efficiency.

We also investigated the voltage dependence of the PL decay, as shown in Figs. 4(a) and 4(b). PL decay times as a function of the applied voltage are summarized in the insets of Fig. 4(a) and 4(b). Figure 3(a) demonstrates a strong dependence of the PL decay times on the applied voltage at $50 \mathrm{~K}$ : while they become longer with increasing forward bias voltages up to $\sim 1 \mathrm{~V}$, above $+1 \mathrm{~V}$, they decrease with a further increase in voltage. These decay times are likely due to the applied electric field below $1 \mathrm{~V}$, compensating for the built-in electric field of $c$-Si solar cells; above $1 \mathrm{~V}$, the applied electric field overcomes the built-in field and activates the carrier extraction from QWs.

In contrast, at $20 \mathrm{~K}$, the PL decay profiles are almost unchanged even under $\pm 1 \mathrm{~V}$ applications. Such a significant difference indicates that the thermal activation process is necessary to extract the carrier from QWs even under strong electric field applications. These results show that the electrons confined in the type-II band offsets can be thermally excited and then extracted by an applied electric field.

Hereafter, we discuss the carrier extraction dynamics in the Ge/Si QWs based on the results of the temperature and voltage dependence of PL decay time, as shown in Fig. 5. At 20 $\mathrm{K}$, the carrier separation rate remains almost unchanged, which can be explained by less thermal excitation because the carriers are confined to the Si layers, as shown in the inset of Fig. 3(b), and cannot overcome the band offset at Ge/Si heterostructures. In contrast, at $50 \mathrm{~K}$ 
the carriers are thermally excited to a higher energy state, and they can then move across the Ge/Si QW layers by electric fields. At the forward bias condition below $1 \mathrm{~V}$, the carrier extraction rate is primarily determined by the electric field: $\Gamma \propto\left|V-V_{\text {built-in }}\right|$, where $V_{\text {built-in }}$ is the built-in potential owing to the $p-i-n$ junction. From this result, the built-in voltages in $c$-Si solar cells were estimated to be $\sim 1 \mathrm{~V}$, which corresponds well to the band gap energy of the Ge/Si QWs. Such correspondence appeared at low temperature is explained well by the conventional $p-n$ junction model. Therefore, our findings indicate that, below $1 \mathrm{~V}$, the thermally activated electrons can be extracted by an applied electric field, and that electrons probably does not limit the performance of photo carrier transportation. In contrast, electrons play a primary role also in dark current generation above $40 \mathrm{~K}$. Then, for highly efficient nanostructure-based solar cells, the dark current generation from electrons would need to be reduced by large band offsets.

Finally, above $1 \mathrm{~V}$, the decay rate increased again but the change was smaller than that observed in the range of $0-1 \mathrm{~V}$. This implies that the carrier separation rate is somehow modified in this voltage range. One possible explanation is that the applied electric field might be screened by electron carriers in this voltage range because the dark current was significantly increased in the range, as can be seen in Fig. 2(b). Another possible explanation is that carrier multiplication occurs under a stronger electric field [24]. To support this speculation, the carrier density dependence of the carrier separation rates needs to be clarified.

\section{Conclusions}

We studied the carrier extraction mechanism from Ge/Si QWs in $c$-Si solar cells and found that the carrier separation dynamics depend on the temperature and the applied electric field, which can be explained by a simple model including the thermal activation process and the 
applied electric field. Our findings indicate that electrons probably does not limit the performance of photo carrier transportation and that the optimum design of the type-II band offset at the $\mathrm{Ge} / \mathrm{Si}$ interface is likely to be a critical issue for suppressed dark current generation.

\section{Acknowledgment}

This work was supported by the JST ALCA program. T.T. was supported by the JST PRESTO program. 


\section{References}

[1] W. Shockley and H. J. Queisser, J. Appl. Phys. 32 (1960) 510.

[2] K. Barnham, I. Ballard, J. Barnes, J. Connolly, P. Griffin, B. Kluftinger, J. Nelson, E. Tsui, and A. Zachariou, Appl. Surf. Sci. 113 (1997) 722.

[3] V. Aroutiounian, S. Petrosyan, A. Khachatryan, and K. Touryan, J. Appl. Phys. 89 (2001) 2268.

[4] A. J. Nozik, Physica E 14 (2002) 115.

[5] P. G. Linares, A. Martí, E. Antolín, C. D. Farmer, Í. Ramiro, C. R. Stanley, and A. Luque, Solar Energy Materials and Solar Cells 98 (2012) 240.

[6] A. Luque, A. Martí, and C. Stanley, Nature Photon. 6 (2012) 146.

[7] A. Ross and A. J. Nozik, J. Appl. Phys. 53 (1982) 3813.

[8] G. J. Conibeer, C. -W. Jiang, D. König, S. Shrestha, T. Walsh, and M. A. Green, Thin Solid Films 516 (2008) 6968.

[9] D. König, Y. Takeda, and B. Puthen-Veettil, Appl. Phys. Lett. 101 (2012) 153901.

[10] Y. Takeda and T. Motohiro, Jap. J. Appl. Phys. 51 (2012) 10ND03.

[11]K. Brunner, Rep. Prog. Phys. 65 (2002) 27.

[12] Y. K. Kuo, Y. K. Lee, Y. S. Ge, S. Ren, J. E. Roth, T. I. Kamins, D. A. B. Miller, and J. S. Harris, Nature 437 (2005) 1334.

[13]T. Tayagaki, S. Fukatsu, and Y. Kanemitsu, Phys. Rev. B 79 (2009) 041301(R).

[14]J. J. Zhang, G. Katsaros, F. Montalenti, D. Scopece, R. O. Rezaev, C. Mickel, B. Rellinghaus, L. Miglio, S. De Franceschi, A. Rastelli, and O. G. Schmidt, Phys. Rev. Lett. 109 (2012) 085502. 
[15]A. Alguno, N. Usami, T. Ujihara, K. Fujiwara, G. Sazaki, K. Nakajima, and Y. Shiraki, Appl. Phys. Lett. 83 (2003) 1258

[16]A. M. Kechiantz, L. M. Kocharyan, and H. M. Kechiyants, Nanotechnology 18 (2007) 405401.

[17]N. Usami, W. Pan, T. Tayagaki, S. T. Chu, J. Li, T. Feng, and T. Kiguchi, Nanotechnology 23 (2012)185401.

[18]T. Tayagaki, N. Usami, W. Pan, Y. Hoshi, K. Ooi, and Y. Kanemitsu, Appl. Phys. Lett. $101(2012) 133905$

[19]T. Tayagaki, N. Usami, and Y. Kanemitsu, Jpn. J. Appl. Phys. 51 (2012) 10NE24.

[20]H. Sunamura, N. Usami, Y. Shiraki, and S. Fukatsu, Appl. Phys. Lett. 66 (1995) 3024.

[21]T. Tayagaki, K. Ueda, S. Fukatsu, and Y. Kanemitsu, J. Phys. Soc. Jpn 81 (2012) 064712.

[22]D. J. Robbins, L. T. Canham, S. J. Barnett, A. D. Pitt, and P. Calcott, J. Appl. Phys. 71 (1992) 1407.

[23]R. O. Rezaev, S. Kiravittaya, V. M. Fomin, A. Rastelli, and O. G. Schmidt, Phys. Rev. B $82(2010) 153306$.

[24]T. Tayagaki, S. Fukatsu, and Y. Kanemitsu, Phys. Status Solidi C 8 (2011) 1049. 


\section{Figure Captions}

Fig. 1. (color online) (a) Schematic illustration of $c$-Si solar cells with multi-stacked $\mathrm{Ge} / \mathrm{Si}$ QWs. (b) Schematic energy diagram of $c$-Si $p$-i-n junction with Ge/Si QWs.

Fig. 2. (color online) (a) Photoluminescence (PL) spectra of Ge/Si quantum wells (QWs) in $c$-Si solar cells measured at $20 \mathrm{~K}(V=0 \mathrm{~V}), 50 \mathrm{~K}(V=0 \mathrm{~V})$, and $50 \mathrm{~K}(V=+1 \mathrm{~V})$. The arrow indicates the detected wavelength for PL decay measurements. (b) Current density $(J)$-voltage ( $V$ ) characteristics of the $c$-Si solar cell with Ge/Si QWs measured at 20, 50, and $300 \mathrm{~K}$.

Fig. 3. (color online) (a) Temporal changes in the PL intensity at $1200 \mathrm{~nm}$ measured at different temperatures. Inset: Temperature dependence of the PL decay time. (b) Temperature dependence of the PL decay rate. The broken curve shows the fitted results. Inset: Schematic energy diagram in the Ge/Si QWs in $c$-Si solar cells.

Fig. 4. (color online) PL decay profiles measured with varying applied voltage: (a) $-1 \mathrm{~V}$ (broke curves), $0 \mathrm{~V}$ (solid curves), $0.6 \mathrm{~V}$ (triangles), and $1 \mathrm{~V}$ (circles) for $50 \mathrm{~K}$. (b) -1, 0, and 1 V for 20 K. Inset: PL decay time as a function of the applied voltage.

Fig. 5. (color online) PL decay rates as functions of the applied voltage measured at 20 (triangles) and 50 (circles) $\mathrm{K}$. The dotted line is a guide to the eye. 


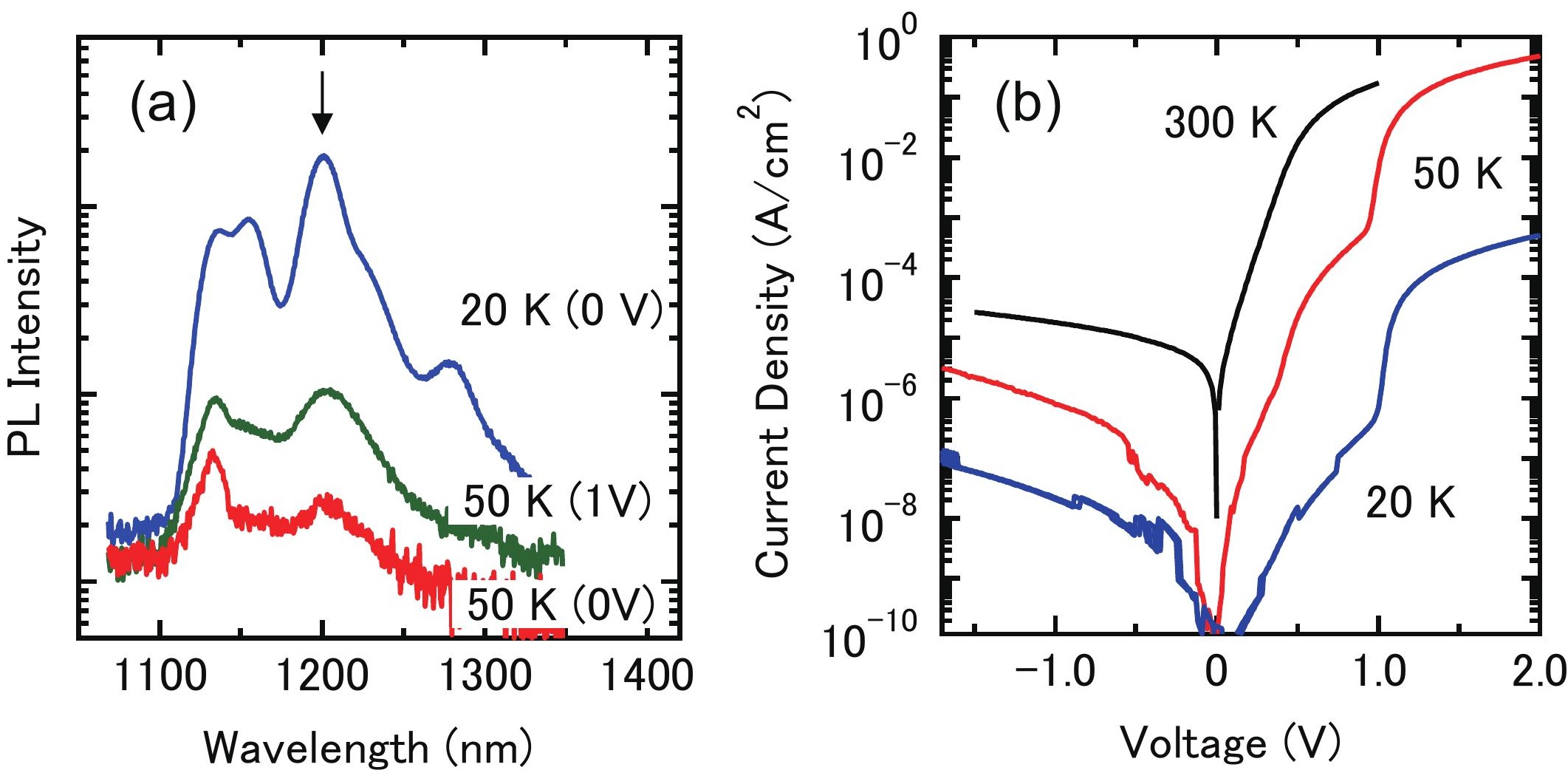



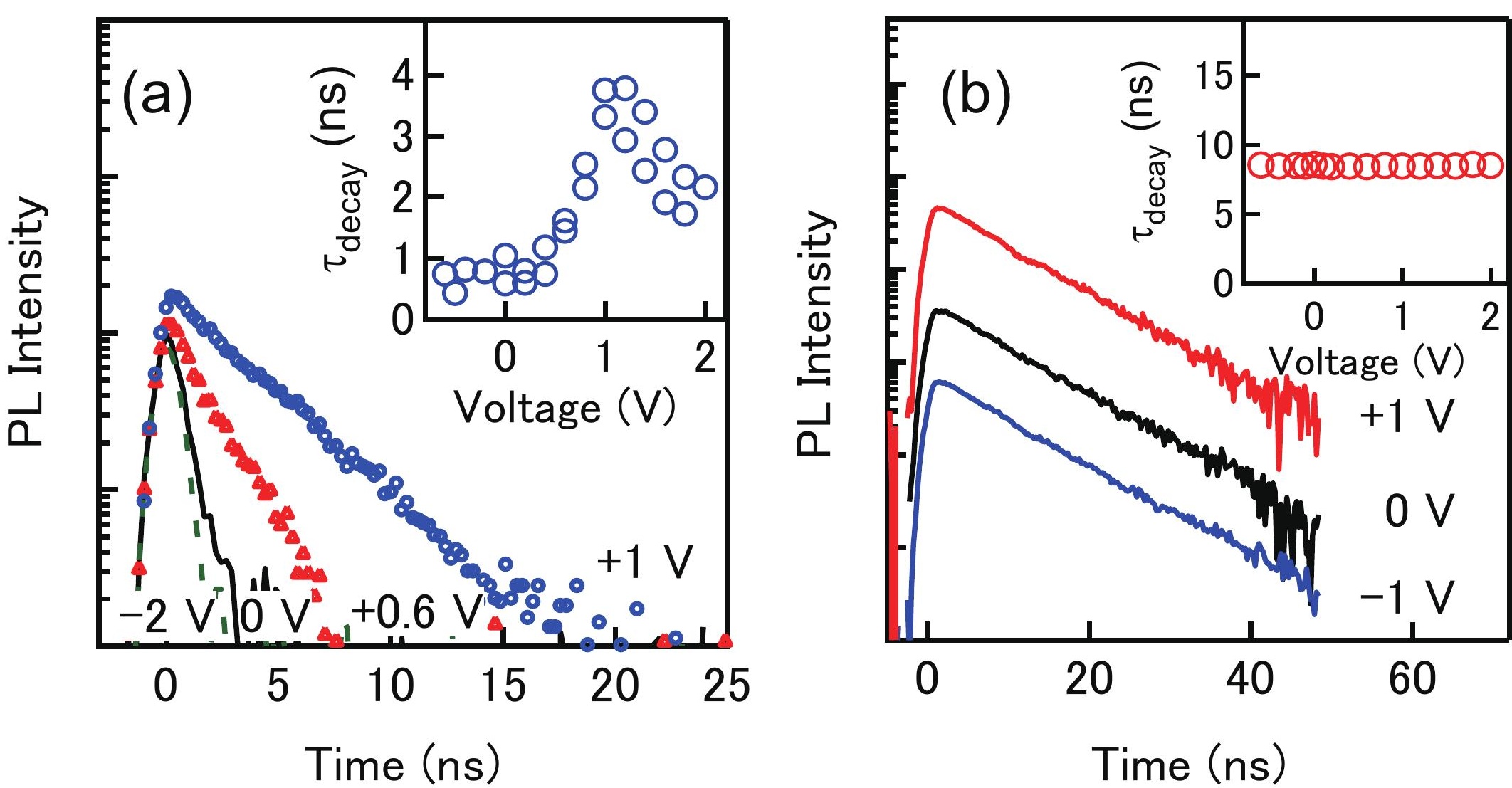


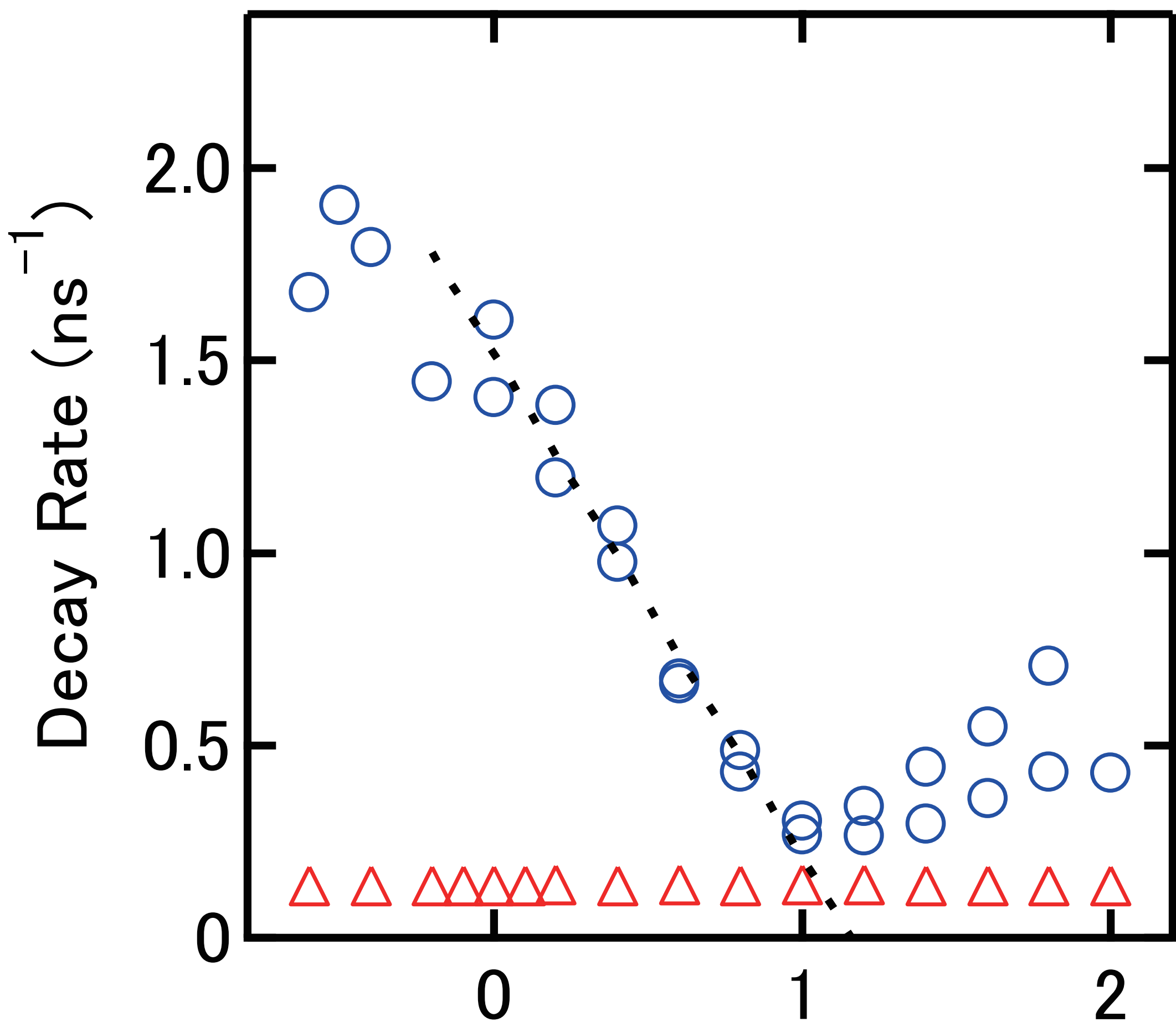

\section{Voltage (V)}

